\title{
Monolayer Characteristics of an N-Acylated Ethanolamine at the Air/ Water Interface
}

\author{
G. Brezesinski, ${ }^{\dagger}$ B. Dobner, ${ }^{\ddagger}$ C. Stefaniu, ${ }^{\dagger}$ and D. Vollhardt ${ }^{*,+}$ \\ ${ }^{\dagger}$ Max Planck Institute of Colloids and Interfaces, D-14424 Potsdam/Golm, Germany \\ ${ }^{\ddagger}$ Institute of Pharmacy, University Halle-Wittenberg, D-06120 Halle, Saale, Germany
}

ABSTRACT: The main characteristics, such as surface pressure-area $(\pi-A)$ isotherms, morphology of the condensed phase domains, lattice structure of the condensed phase, and the existence of hydrogen bonds $(-\mathrm{NH} \cdots \mathrm{O}=\mathrm{C}-)$ of the monolayers of a highly purified $\mathrm{N}$-acylated ethanolamine $\left(\mathrm{C}_{13} \mathrm{H}_{27}-\mathrm{CO}-\mathrm{NH}-\mathrm{C}_{2} \mathrm{H}_{4} \mathrm{OH}\right.$, TDAHA $)$, are determined. At temperatures measured between 10 and $36^{\circ} \mathrm{C}$, the characteristics of the surface pressure - area $(\pi-A)$ isotherms, which indicate the main first-order phase transition from the fluid phase to the condensed phase, are similar to those of usual amphiphiles. However, at temperatures of $\leq 10^{\circ} \mathrm{C}$, a second inflection point whose transition pressure increases strongly with increasing temperature proves the existence of a second first-order phase transition between two condensed phases. The entropy change of this second transition is small with only $10 \%$ of that observed for the main phase transitions at the same temperature. The fractal-like shaped condensed phase

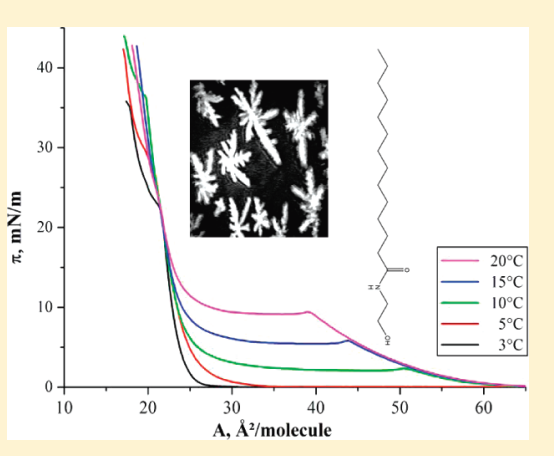
domains have a limited tendency to branching and are characterized by the absence of an inner texture in the homogeneously reflecting domains. The characteristic features of the two-dimensional lattice structure are obtained by GIXD studies. An oblique lattice is formed after the main phase transition with a cross-sectional area of $19.3 \AA^{2}$, whereas after the second phase transition an orthorhombic $\mathrm{L}_{2}^{\prime}$ phase arises accompanied by a drastic decrease of the molecular tilt angle. The IRRAS experiments confirm the existence of an extended hydrogen bonding network $(-\mathrm{NH} \cdots \mathrm{O}=\mathrm{C}-$ ) in the TDAHA monolayers by the positions of the amide bands and the transition from an oblique into an orthorhombic phase by the split of the $\mathrm{CH}_{2}$ deformation band.

\section{INTRODUCTION}

$N$-Acylethanolamines (NAEs) are naturally occurring amphiphiles that are present in a wide variety of animals, plants, and microbes. ${ }^{1,2}$ Therefore, and because of a number of interesting biological and medicinal properties, $N$-acylethanolamines attracted particular attention. Obviously, their production in a variety of organisms increases dramatically as a response to stress, such as injury in animals and dehydration in plants. ${ }^{1,3} \mathrm{~N}$-acylethanolamines have also been reported to accumulate in different types of human tumors and in the surrounding normal tissue assuming that their accumulation in tumors might be due to their production by the adjacent tissues to fight the cancerous growth. ${ }^{4}$ $\mathrm{N}$-acylethanolamines reveal anti-inflammatory, antibacterial, and antiviral properties, which may have considerable therapeutic potential. ${ }^{1}$ Striking biological properties have been found dependent on specific unsaturated and saturated acyl chains of different length. For example, in the case of an unsaturated acyl chain, $\mathrm{N}$-arachidonylethanolamine acts as an endogenous ligand for cannabinoid receptor, ${ }^{5}$ reduces sperm fertilizing capacity, ${ }^{6}$ and inhibits gap junction conductance. ${ }^{7} \mathrm{~N}$-oleoylethanolamine is a potent inhibitor of ceramidase, ${ }^{8}$ whereas in the case of a saturated acyl chain, $N$-palmitoylethanolamine acts as an agonist for the cannabinoid receptor-type 2 , and $N$-myristoylethanolamine and $\mathrm{N}$-lauroylethanolamine are secreted into the culture medium of tobacco cells in response to stress. ${ }^{9}$ Correspondingly, studies of $\mathrm{N}$-acylethanolamines have been carried out to understand the metabolism as well as their phase behavior and interactions with other membrane lipids such as DPPC, DMPC, and DMPE and proteins. $3,10,11$

Potential applications of $N$-acylethanolamines in developing liposomal drug delivery systems and as therapeutic agents have been suggested, as they are able to stabilize liposomes, even in the presence of human serum. ${ }^{12-14}$ Therefore, the interaction of several $N$-acylethanolamines with dipalmitoylphosphatidylcholine (DPPC) multilamellar vesicles was studied. ${ }^{14}$

Specific information has been obtained about the effects of hydrogen bonding systems, dipole interactions, and sterical hindrance on packing in $3 \mathrm{D}$ crystals of selected $\mathrm{N}$-acylethanolamines and $N$-acylpropanolamines. ${ }^{15-17}$

In general, the role of amide and amine groups is of special interest because these groups are an integral part of the general structure of sphingolipids. ${ }^{18,19}$ Sphingolipids, consisting of longchain amino alcohols (sphingosine or dihydrosphingosine) linked by an amide bond to a fatty acid, and their metabolites are involved in many vital biological processes including differentiation, cellular senescence, apoptosis, and proliferation. ${ }^{20}$

For understanding the role of amide containing amphiphiles in inherently complex biological processes, Langmuir monolayers can be used as simple model systems. Therefore, in previous

Received: January 26, 2011

Published: April 01, 2011 


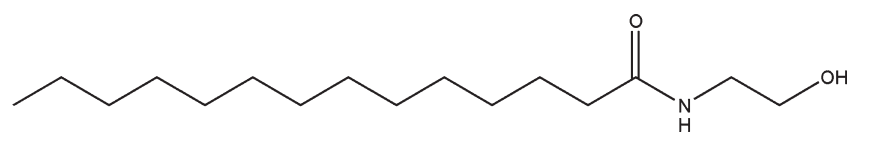

Figure 1. Chemical structure of $N$-myristoylethanolamine (tetradecanoic acid-(2-hydroxyethyl)amide, TDAHA).

papers, the main monolayer characteristics of various tailored amphiphiles, the headgroup of which consists of an acid amide group and one or two hydroxyl groups separated by one or more (two or three) methylene groups, were studied. $^{21-31}$ The domain morphology of this type of amphiphiles is generally crystalline, despite notable differences in the domain textures.

According to the biological role and the therapeutic potential of $\mathrm{N}$-acylethanolamines, the objective of a previous work was to study the effect of the position of the substituents at the amide group on the major monolayer characteristics. For this purpose, the monolayer characteristics of two similar amphiphiles $\mathrm{N}$-myristoylethanolamine $\left(\mathrm{C}_{13} \mathrm{H}_{27}-\mathbf{C O}-\mathrm{NH}-\mathrm{C}_{2} \mathrm{H}_{4} \mathrm{OH}\right.$; HETA $)$ and $N$-tridecyl$\beta$-hydroxypropionic acid amide $\left(\mathrm{C}_{13} \mathrm{H}_{27}-\mathrm{NH}-\mathrm{CO}-\mathrm{C}_{2} \mathrm{H}_{4} \mathrm{OH}\right.$; THPA) were compared in ref 32 . The only difference in the chemical structure is the different position of the two substituents at the amide group. Renewed synthesis and careful analysis of the two amphiphiles have shown that the $N$-myristoylethanolamine (HETA) sample used in ref 32 contained a second amphiphilic component as an impurity. The possible effect of surface-active impurities on the characteristics of the main component is a known phenomenon. The most representative example of such an effect is the dominance of dodecanol traces in the characteristics of the main component sodium dodecylsulfate. ${ }^{33,34}$

In the present study, $\mathrm{N}$-myristoylethanolamine $\left(\mathrm{C}_{13} \mathrm{H}_{27}-\right.$ $\mathrm{CO}-\mathrm{NH}-\mathrm{C}_{2} \mathrm{H}_{4} \mathrm{OH}$, tetradecanoic acid-(2-hydroxyethyl)amide, TDAHA, Figure 1) of high purity has been synthesized, and the main characteristics of its Langmuir monolayers, such as surface pressure-area $(\pi-A)$ isotherms, morphology of the condensed phase domains, lattice structure of the condensed phase, and existence of hydrogen bonds $(-\mathrm{NH} \cdots \mathrm{O}=\mathrm{C}-)$, are presented.

\section{EXPERIMENTAL SECTION}

Materials. Synthesis of N-Myristoylethanolamine (Tetradecanoic Acid-(2-hydroxyethyl)amide, TDAHA). A solution of $0.01 \mathrm{~mol}$ $(2.47 \mathrm{~g})$ of tetradecanoic acid chloride was slowly dropped to a mixture of $0.05 \mathrm{~mol}(3.0 \mathrm{~g})$ of 2-aminoethanol in $40 \mathrm{~mL}$ of dry chloroform at $0{ }^{\circ} \mathrm{C}$ under stirring. The mixture was brought to room temperature and then allowed to stir for a further $4 \mathrm{~h}$ at that temperature. The mixture was washed twice with $40 \mathrm{~mL}$ of water, and afterward, the organic layer was separated. After drying over sodium sulfate, the solvent was evaporated and the crude product purified by recrystallization from acetone. Yield: $2.29 \mathrm{~g}$ (84.5\%), white crystals. mp: $90-91{ }^{\circ} \mathrm{C}$. ESI-MS: $294.2\left(65 \%,[\mathrm{M}+\mathrm{Na}]^{+}\right)$, $565.1\left(100 \%,\{2 \mathrm{x}[\mathrm{M}]+\mathrm{Na}\}^{+}\right)$. Elemental analysis: Calcd: $\mathrm{C}$ 70.80, H 12.25, N 5.16. Found: C 70.74, H 12.33, N 5.12. ${ }^{1} \mathrm{H}$ $\operatorname{NMR}\left(400 \mathrm{MHz}, \mathrm{CDCl}_{3}\right): \delta=0.84\left(\mathrm{t}, 3 \mathrm{H},\left[-\mathrm{CH}_{3}\right]\right), 1.17-1.38$ $\left(\mathrm{m}, 2 \mathrm{H}\right.$, [chain] ), 1.56-1.70 (m, 2H, [-NH-CO- $\left.\left.\mathrm{CH}_{2} \mathrm{CH}_{2}-\right]\right)$, $2.2\left(\mathrm{t}, 2 \mathrm{H},\left[-\mathrm{NH}-\mathrm{CO}-\mathrm{CH}_{2} \mathrm{CH}_{2}-\right]\right), 3.38-3.42(\mathrm{~m}, 2 \mathrm{H}$, $\left.\left[\mathrm{HO}-\mathrm{CH}_{2} \mathrm{CH}_{2}-\mathrm{NH}-\mathrm{CO}-\right]\right), 3.7\left(\mathrm{t}, 2 \mathrm{H},\left[\mathrm{HO}-\mathrm{CH}_{2} \mathrm{CH}_{2}-\right.\right.$ $\mathrm{NH}-\mathrm{CO}-$ ]) ppm.
The spreading solvent used was chloroform (p.a. grade, Baker, Holland). Ultrapure water produced by "Purelab Plus" was used as a subphase.

Surface Pressure Measurements and Brewster Angle Microscopy. An experimental setup consisting of a self-made computer interfaced film balance coupled with a Brewster angle microscope (BAM1+, NFT, Göttingen, Germany) was used to measure the equilibrium surface pressure $(\pi-A)$ isotherms at a compression rate of $\leq 10 \AA^{2} /$ (molecule min). ${ }^{32}$ According to the Wilhelmy method, the surface pressure was measured with a roughened glass plate with an accuracy of $\pm 0.1 \mathrm{mN} \mathrm{m}^{-1}$ and the area per molecule with $\pm 0.5 \AA^{2}$.

Detailed information about the BAM method is given elsewhere (see, e.g., refs $35-37$ and references therein). The lateral resolution of the BAM1+ was approximately $4 \mu \mathrm{m}$.

$X$-ray Diffraction Measurements ${ }^{38-41}$. The lateral structures in the monolayer at the air/water interface were investigated using grazing incidence X-ray diffraction (GIXD) measurements at the BW1 beamline, HASYLAB (Hamburg, Germany). A monochromatic X-ray beam $(\lambda=1.304 \AA)$ strikes the water surface at a grazing incidence angle of $\alpha_{i}=0.85 \alpha_{c}$ (where $\alpha_{c}=0.13^{\circ}$ is the critical angle for total reflection of the X-ray beam at the water surface), illuminating roughly a $2 \times 50 \mathrm{~mm}^{2}$ monolayer surface in a thermostatted Langmuir film balance placed in a hermetically closed container filled with helium. During the course of the experiment, a slow lateral movement of the trough is used in order to avoid sample damage by the strong X-ray beam. A MYTHEN detector system (PSI, Villigen, Switzerland) measures the diffracted signal and is rotated to scan the in-plane $Q_{x y}$ component values of the scattering vector. The vertical strips of the MYTHEN measure the out-of-plane $Q_{z}$ component of the scattering vector between 0.0 and $0.75 \AA^{-1}$. The diffraction data consist of Bragg peaks at diagnostic $Q_{x y}$ values. These peaks are calculated by summing the diffracted intensity at each in-plane $Q_{x y}$ value over a defined vertical angle or $Q_{z}$ window. The inplane lattice repeat distances $d$ of the ordered structures in the monolayer are calculated from the Bragg peak positions: $d=2 \pi$ / $Q_{x y}$. To access the extent of the crystalline order in the monolayer, the in-plane coherence length $L_{x y}$ is approximated from the full width at half-maximum (fwhm) of the Bragg peaks using $L_{x y} \sim 0.9(2 \pi) /$ fwhm $\left(Q_{x y}\right)$. The diffracted intensity normal to the interface is integrated over the $Q_{x y}$ window containing the diffraction peak to calculate the corresponding Bragg rod. The thickness of the monolayer is estimated from the fwhm of the Bragg rod using $0.9(2 \pi) /$ fwhm $\left(Q_{z}\right)$.

IRRAS Measurements ${ }^{42-45}$. The measurements were performed using an IFS 66 FTIR spectrometer (Bruker, Karlsruhe, Germany) equipped with a liquid nitrogen cooled mercury cadmium telluride detector attached to an external air/water reflection unit (XA - 511, Bruker). The principle of the method and its application to Langmuir films at the air-water interfaces are described in ref 42. A small reference trough and the larger sample trough are alternatively moved into the IR beam path by a shuttling mechanism. The resolution and scanner speed used in all experiments were $8 \mathrm{~cm}^{-1}$ and $20 \mathrm{kHz}$, respectively. The incident IR beam is polarized with a KRS-5 wire grid polarizer. The measured signal represents the ratio of the reflected light from two liquid surfaces: $\mathrm{RA}=-\log [$ (sample reflectivity) $/($ referencez reflectivity $)]=-\log \left(R / R_{0}\right)$ in reflectance-absorbance (RA) units. Spectra were coadded over 400 scans for p-polarized light and over 200 scans for s-polarized light. The two different light polarizations provide information on molecular 


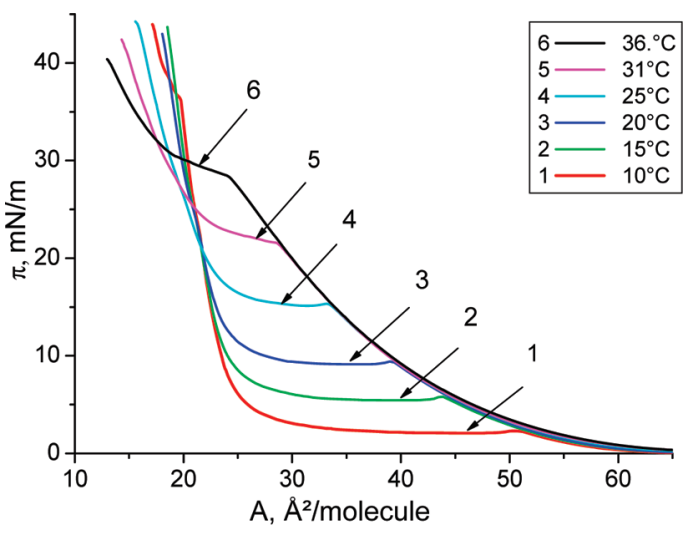

Figure 2. $\pi-A$ isotherms of TDAHA monolayers spread on water and measured in the temperature range between 10 and $36^{\circ} \mathrm{C}$.

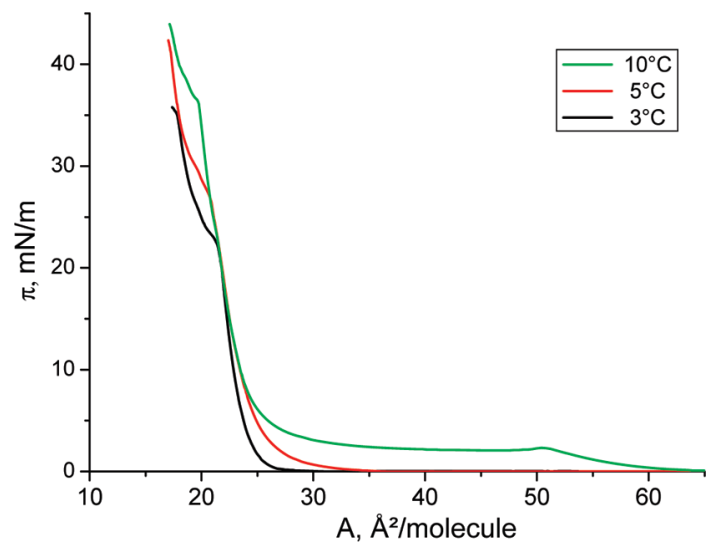

Figure 3. $\pi-A$ isotherms of TDAHA monolayers spread on water and measured in the temperature range between 3 and $10^{\circ} \mathrm{C}$.

orientation with respect to the surface plane of the monolayer. A change in the intensity ratio of $\mathrm{p}$-polarized to s-polarized light ( $\mathrm{p}: \mathrm{s}$ ratio) for a vibrational band indicates a change in the average orientation of the vibration and thus of the molecule.

\section{RESULTS AND DISCUSSION}

The experimental $\pi-A$ isotherms of the TDAHA monolayers spread on pure water and measured at different temperatures in the range between 10 and $36^{\circ} \mathrm{C}$ are shown in Figure 2. In this temperature range, the features of the $\pi-A$ isotherms of TDAHA are those of usual monolayers of amphiphiles. ${ }^{46}$ The isotherms show a sharp inflection at the first-order phase transition point $\left(A_{c}\right)$ from the fluid (liquid-expanded, LE; gaseous, $G$ ) phase to the condensed phase. In the following two-phase coexistence region $\left(A<A_{\mathrm{c}}\right)$, the slope of the isotherm is much lower than that compared to the fluid state, and it becomes nearly horizontal with the decrease in temperature. As usual, the extension of the phase transition region of the TDAHA monolayers decreases as the temperature increases. The $A_{c}$ values indicating the onset of the main phase transition become smaller with increasing temperature. This transition can be seen at temperatures $\geq 7{ }^{\circ} \mathrm{C}$.

The changed slope of the isotherms and their shift to unusual small area values in the condensed part with increasingly higher temperatures shows that the solubility of TDAHA increases

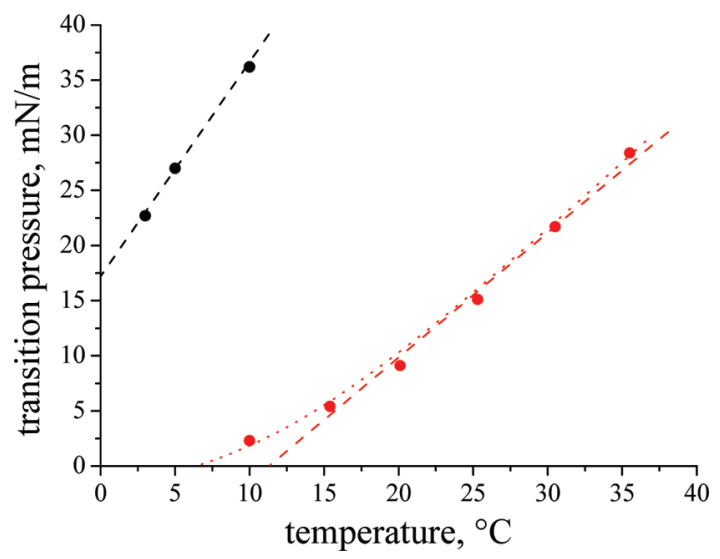

Figure 4. Temperature dependence of the main phase transition pressure $\pi_{\mathrm{c}}$ and the second phase transition between the two condensed phases of TDAHA monolayers spread on water.

with increasing temperature. In this region, a certain amount of the monolayer material is lost during compression. The estimation of the area per molecule in the condensed state at the end of the first-order phase transition is therefore connected with larger error bars.

The $\pi-A$ isotherm measured at the lowest temperature of $10^{\circ} \mathrm{C}$ in Figure 2 shows obviously a second weak inflection point at a high surface pressure of $\sim 36 \mathrm{mN} / \mathrm{m}$, indicating the existence of a second phase transition between two condensed phases. To characterize this second phase transition point in more detail, isotherm measurements have been performed at lower temperatures.

Figure 3 shows three $\pi-A$ isotherms of TDAHA monolayers measured between 10 and $3{ }^{\circ} \mathrm{C}$. The area change after the second inflection point is very small compared with the main phase transition. Such a striking second critical point has been observed in the low temperature isotherms of a very similar amphiphile (3-hydroxy- $\mathrm{N}$-tridecyl propanoic acid amide, $\mathrm{C}_{13} \mathrm{H}_{27}-\mathrm{NH}-$ $\mathrm{CO}-\mathrm{C}_{2} \mathrm{H}_{4} \mathrm{OH}, \mathrm{HTPA}$ ) whose chemical structure is only different from that of TDAHA because of the exchange of the position of the two substituents at the acid amide group..$^{24,32,47}$ It is interesting to note that this second phase transition between two condensed phases of TDAHA monolayers is existent mainly at such low temperatures $\left(\leq 10{ }^{\circ} \mathrm{C}\right)$ where the fluid/condensed phase transition approaches zero pressure. However, in contrast to HTPA, whose transition pressure of the second phase transition is nearly independent of temperature, this second phase transition pressure in TDAHA monolayers is strongly dependent on temperature (Figure 3). The transition pressure increases with increasing temperature. The temperature dependence is with $1.912 \mathrm{mN} /(\mathrm{m} \cdot \mathrm{K})$ stronger compared to that of the main transition pressure (Figure 4). The area change during this second transition is very small. At $10^{\circ} \mathrm{C}$, a $\Delta S$ of $-23 \mathrm{~J} /(\mathrm{mol} \cdot \mathrm{K})$ can be calculated compared with $-219 \mathrm{~J} /(\mathrm{mol} \cdot \mathrm{K})$ for the transition from the fluid (LE, G) to the condensed phase.

The characteristics of the experimental $\pi-A$ isotherms of the TDAHA monolayers deviate essentially from those obtained for HETA in ref 32. The renewed synthesis procedure of TDAHA has revealed that a second amphiphilic N,O-diacyl compound of ethanolamine was present as an impurity and affected strongly the interfacial characteristics of HETA.

The two-dimensional Clapeyron equation representing a onecomponent approximation has been used for calculating the 


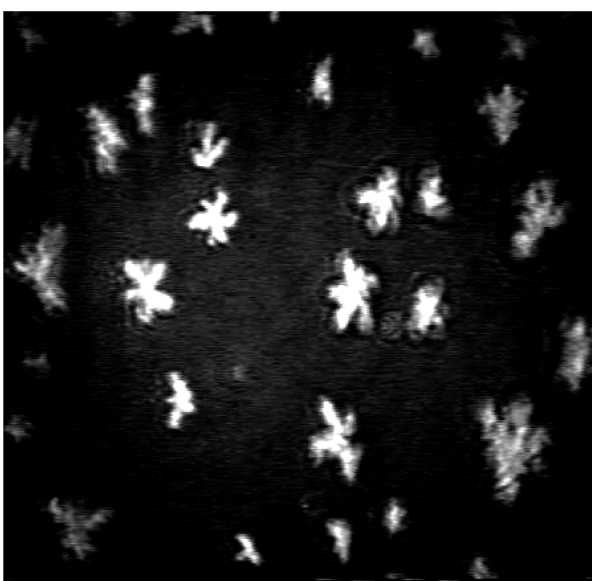

a)

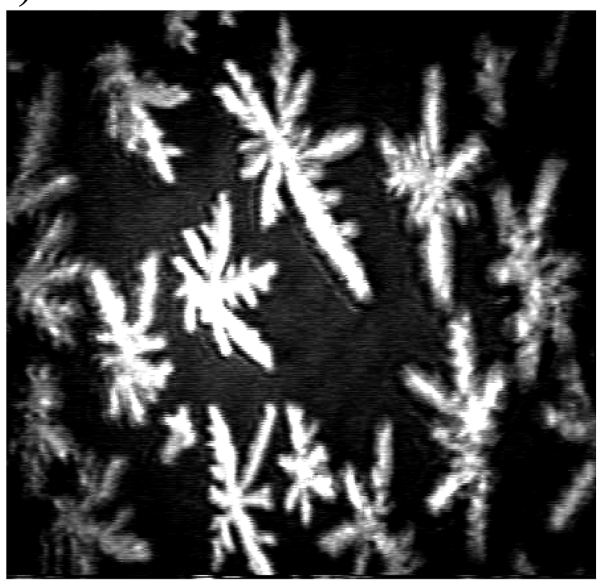

c)

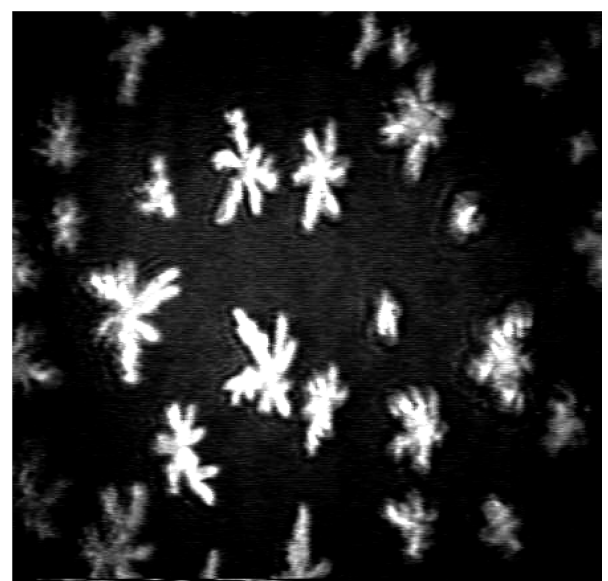

b)

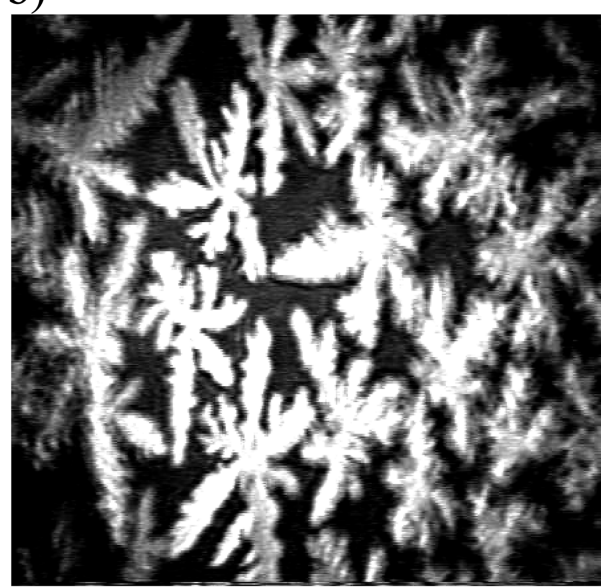

d)

Figure 5. Growth steps of typical TDAHA domains formed after the main phase transition point within the fluid/condensed transition region. Compression rate: $1 \AA^{2} /($ molecule $\min ), T=15^{\circ} \mathrm{C}$.

enthalpy change $\Delta H$ of the phase transition

$$
\Delta H=\left(A_{\mathrm{c}}-A_{\mathrm{e}}\right) T \frac{\mathrm{d} \pi_{\mathrm{t}}}{\mathrm{d} T}
$$

where $A_{\mathrm{e}}$ is the molecular area at the onset of the phase transition at the surface pressure $\pi_{\mathrm{t}}$ and $A_{\mathrm{c}}$ is the area of the condensed phase at this pressure. The slope of the straight line of the $\mathrm{d} \pi_{\mathrm{c}} / \mathrm{d} T$ curve in Figure 4 has been used, and the small deviation from linearity near zero pressure is not considered. Negative $\Delta H$ and $\Delta S$ values are obtained, indicating the exothermic nature of this transition on compression of the monolayer and an increase in the ordering of the system. The absolute $\Delta S$ and $\Delta H$ values increase as the temperature decreases, indicating that the ordering of the condensed phase increases at lower temperatures. The critical temperature of $41.1{ }^{\circ} \mathrm{C}$, compared to $48.6^{\circ} \mathrm{C}$ determined for HTPA, ${ }^{32,48}$ for the main phase transition results from the extrapolation to $\Delta S=0$. Above this temperature, the monolayer cannot form anymore a condensed state by compression.

Formation and growth of the condensed phase domains within the fluid/condensed phase transition region has been visualized by BAM experiments (Figure 5). The bright condensed phase domains are surrounded by the fluid phase of lower density and thus lower reflectivity. After the fluid/condensed phase transition point $\pi_{\mathrm{c}}$ at the beginning of the plateau region, first small, irregularly shaped domains are formed (Figure 5a). Some rather thick arms growing from the center in different directions are seen. The following growth stages reveal not only differences in the averaged thickness of the main axes but also a limited tendency to branching (Figure $5 \mathrm{~b}-\mathrm{d}$ ). The fractal nature of the homogeneously reflecting domain shape and the absence of an inner texture of the domains become obvious. These characteristics indicate a certain fluidity of the condensed phase.

Grazing incidence X-ray diffraction (GIXD) has been applied to obtain information about the two-dimensional structure of the condensed TDAHA monolayer. The diffraction studies were performed on water at $5{ }^{\circ} \mathrm{C}$ and at different lateral pressures. Figure 6 shows selected contour plots of the corrected X-ray diffraction intensities as a function of the in-plane scattering vector component $Q_{x y}$ and the out-of-plane scattering vector component $Q_{z}$ obtained at different lateral pressures. The three Bragg peaks observed at all pressures between the first and the second phase transition show that the structure of the condensed monolayer phase in this region is oblique. The alkyl chains are strongly tilted in a nonsymmetry direction. Above the second transition pressure, the condensed monolayer phase changes to an orthorhombic structure with NNN (next nearest neighbors) tilted chains.

The corresponding lattice parameters of the TDAHA monolayer measured at $5{ }^{\circ} \mathrm{C}$ are listed in Table 1 .

Calculating the lattice parameters from the X-ray data for these two phases shows that the cross-sectional area, $A_{0}$, amounts to 


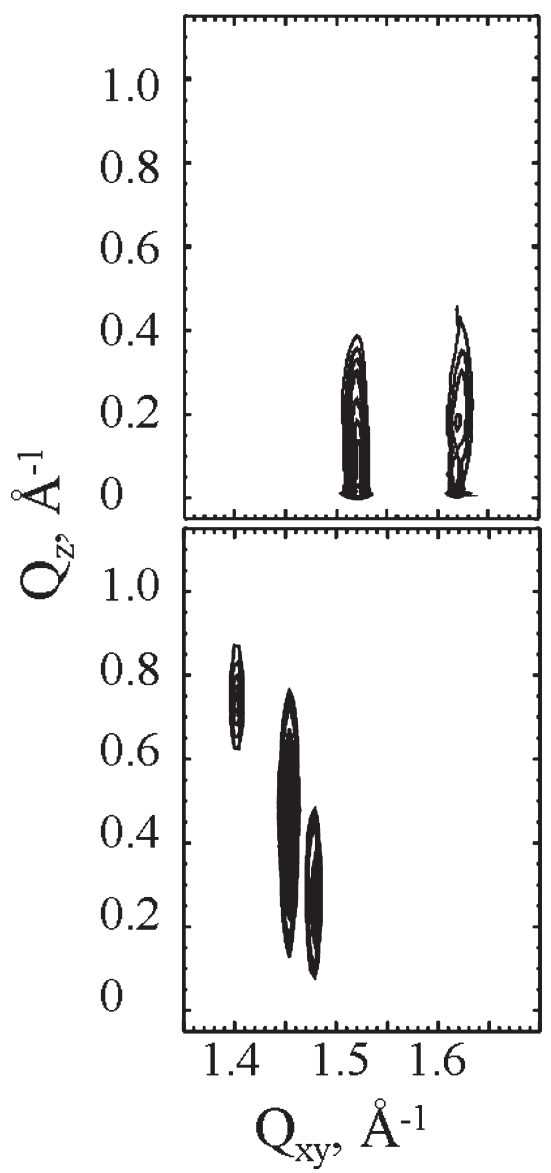

Figure 6. GIXD contour plots of the corrected diffraction intensities as a function of the in-plane $Q_{x y}$ and out-of-plane $Q_{z}$ components of the scattering vector for TDAHA monolayers at $5{ }^{\circ} \mathrm{C}$ and different pressures (bottom, $10 \mathrm{mN} / \mathrm{m}$; top, $30 \mathrm{mN} / \mathrm{m}$ ).

Table 1. Lattice Parameters $a, b, c$ and $\alpha, \beta, \gamma$ of the Unit Cell, Lattice Distortion, Chain Tilt $t$ from the Surface Normal, InPlane Area $A_{x y}$ per Chain, Cross-Sectional Area $A_{0}$ of TDAHA Monolayers at $5{ }^{\circ} \mathrm{C}$, and Different Lateral Pressures $\pi$

\begin{tabular}{ccccccc}
\multicolumn{6}{c}{$a(\AA)$} & $\alpha(\mathrm{deg})$ \\
& $b(\AA)$ & $\beta(\mathrm{deg})$ & & & & \\
$\pi(\mathrm{mN} / \mathrm{m})$ & $c(\AA)$ & $\gamma(\mathrm{deg})$ & distortion & $t(\mathrm{deg})$ & $A_{x y}\left(\AA^{2}\right)$ & $A_{0}\left(\AA^{2}\right)$ \\
\multirow{2}{*}{4} & 4.885 & 123.1 & 0.06825 & 29.3 & 22.0 & 19.2 \\
& 5.081 & 119.4 & & & & \\
& 5.179 & 117.4 & & & & \\
10 & 4.891 & 122.4 & 0.05310 & 27.8 & 21.8 & 19.3 \\
& 5.037 & 119.6 & & & & \\
& 5.120 & 117.9 & & & & \\
20 & 4.883 & 122.3 & 0.04910 & 26.3 & 21.6 & 19.4 \\
& 5.021 & 119.6 & & & & \\
& 5.093 & 118.1 & & & & \\
30 & 4.887 & 115.5 & 0.08839 & 7.4 & 18.9 & 18.8 \\
& 4.580 & 122.2 & & & & \\
& 4.580 & 122.2 & & & & \\
\hline
\end{tabular}

19.3 $\AA^{2}$ in the oblique phase and decreases to $18.8 \AA^{2}$ in the orthorhombic phase. The tilt is in NNN direction and the

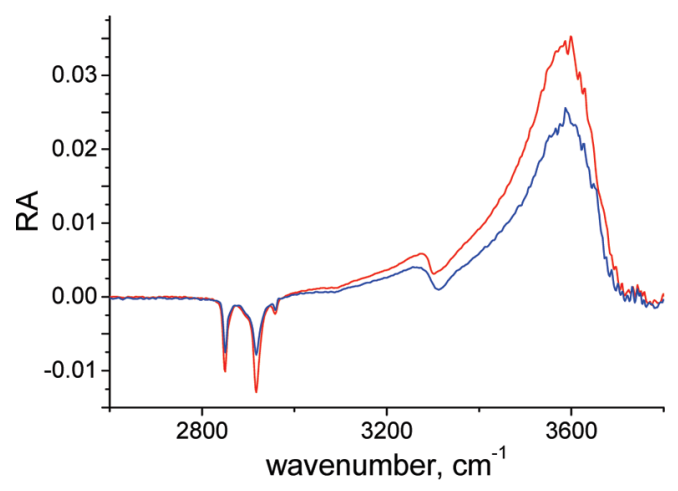

Figure 7. IRRA spectra of TDAHA monolayer at $10 \mathrm{mN} / \mathrm{m}$ (blue line) and at $30 \mathrm{mN} / \mathrm{m}$ (red line) measured at $5{ }^{\circ} \mathrm{C}$ with p-polarized light at an angle of incidence of $40^{\circ}$ in the $\mathrm{OH}$ and $\mathrm{CH}$ stretching vibration regions.

distortion in NN (nearest neighbor) direction; therefore, this orthorhombic phase can be named $\mathrm{L}_{2}^{\prime}$. The cross-sectional areas have typical values for a phase with ordering of the alkyl chain backbones. ${ }^{40}$ The dimensions of the projected rectangular unit cell containing two molecules are $4.887 \times 7.683 \AA^{2}$. Such a dense molecular packing is a fingerprint of the herringbone ( $\mathrm{HB}$ ) arrangement, usually found for the packing of hydrocarbon chains in bulk crystals. ${ }^{47}$

The tilt angle with respect to the surface normal, $t$, decreases only marginally with increasing pressure $\left(0.18^{\circ}\right.$ per $\left.\mathrm{mN} / \mathrm{m}\right)$ in the oblique phase. The transition pressure into a nontilted phase can be calculated by plotting of $1 / \cos (t)$ as a function of the lateral pressure and extrapolating toward $1 .{ }^{38}$ The nontilted phase can be expected only at unrealistically high pressure values of around $80 \mathrm{mN} / \mathrm{m}$. The transition into the orthorhombic $\mathrm{L}_{2}^{\prime}$ phase leads to a drastic decrease of the tilt angle by almost $20^{\circ}$ and a decrease in the cross-sectional area by $2.5 \%$.

The phase properties of the TDAHA monolayers can be fundamentally characterized on the basis of the surface pressure and GIXD results. At temperatures below $10^{\circ} \mathrm{C}$, the monolayers reveal two first-order phase transitions in the $\pi-A$ isotherms. The main phase transition is connected with the transition from the disordered liquid-like state (LE) into a condensed phase which has an oblique lattice structure. The second transition into an orthorhombic structure with NNN (next nearest neighbors) tilted chains at higher pressures is a very weak first-order transition with only $10 \%$ of the entropy change observed for the first first-order main phase transitions at the same temperature. Both transition pressures increase with increasing temperature; therefore, the second transition cannot be seen in the isotherms above $10{ }^{\circ} \mathrm{C}$, since the monolayer stability does not allow the compression above $40 \mathrm{mN} / \mathrm{m}$.

Figure 7 shows the IRRA spectra of the TDAHA monolayer between 2600 and 3800 wavenumbers at two different lateral pressures below and above the observed transition between the two condensed phases. The prominent band at $3580 \mathrm{~cm}^{-1}$ arises from the $\mathrm{OH}$ stretch of water and is a characteristic feature of IRRA spectra. The water $\mathrm{OH}$ stretching vibration present in the reference signal $\left(R_{0}\right)$ is reduced in the reflectivity signal from the monolayer-covered surface $(R)$ because the TDAHA layer replaces a water layer and masks partially the $\mathrm{OH}$ stretching vibration. The result is a strong positive band that is related to the monolayer's effective thickness. The increase in the band intensity at higher pressure (above the phase transition) is attributed 


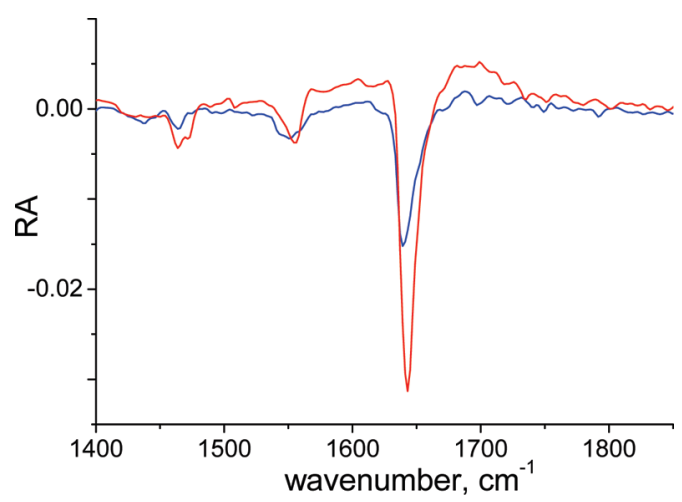

Figure 8. The amide I and II regions as well as the $\mathrm{CH}_{2}$ deformation region of IRRA spectra of TDAHA monolayers at $10 \mathrm{mN} / \mathrm{m}$ (blue line) and at $30 \mathrm{mN} / \mathrm{m}$ (red line) measured at $5{ }^{\circ} \mathrm{C}$ with p-polarized light at an angle of incidence of $45^{\circ}$.

to the effectively thicker TDAHA layer because of the much smaller tilt observed in the GIXD experiments.

The CH stretching vibration region $\left(2840-2975 \mathrm{~cm}^{-1}\right)$ is characterized by two prominent bands (symmetric and asymmetric $\mathrm{CH}_{2}$ stretching vibrations) and one much weaker band (asymmetric $\mathrm{CH}_{3}$ stretching vibration). The position of the $\mathrm{CH}_{2}$ stretching bands shifts slightly to lower wavenumbers from 2850.4 to $2849.7 \mathrm{~cm}^{-1}$ for the symmetric and from 2918.5 to $2917.5 \mathrm{~cm}^{-1}$ for the asymmetric stretching vibration on compression from 10 to $30 \mathrm{mN} \cdot \mathrm{m}^{-1}$. The $\mathrm{CH}_{3}$ asymmetric stretching does not change the position on compression and is located at $2958.3 \mathrm{~cm}^{-1}$. The observed band positions clearly indicate a well-packed condensed monolayer with all-trans conformation of the alkyl chains. The slight shift of the $\mathrm{CH}_{2}$ stretching bands corresponds well to the observed tighter packing in the orthorhombic phase (herringbone packing) compared to the oblique phase at lower pressures. The intensity of the bands measured with p- and s-polarized light increases markedly only above the oblique- $\mathrm{L}_{2}{ }^{\prime}$ transition in accordance with the higher packing density in the $\mathrm{L}_{2}^{\prime}$ phase. The dichroic ratio, $A_{\mathrm{p}} / A_{\mathrm{s}}$, also increases on compression in accordance with the observed change of the tilt angle.

The IRRAS experiments provide also information about the existence of hydrogen bonds $(-\mathrm{NH} \cdots \mathrm{O}=\mathrm{C}-$ ) in the TDAHA monolayer. It is well-known that $\mathrm{H}$-bonds shift the bands of the stretching vibrations $v(\mathrm{NH})$ and $v(\mathrm{C}=\mathrm{O})$ to lower wavenumbers and that of the deformation peak $\delta(\mathrm{NH})$ in the opposite direction.

The HTPA $\left(\mathrm{C}_{13} \mathrm{H}_{27}-\mathrm{NH}-\mathrm{CO}-\mathrm{C}_{2} \mathrm{H}_{4} \mathrm{OH}\right)$ molecules in $\mathrm{CCl}_{4}$ solution showed a single $v(\mathrm{NH})$ band at $3462 \mathrm{~cm}^{-1}$, typical for the free $\mathrm{NH}$ stretching of trans polar heads of secondary amides. ${ }^{48}$ Unfortunately, TDAHA $\left(\mathrm{C}_{13} \mathrm{H}_{27}-\mathrm{CO}-\right.$ $\left.\mathrm{NH}-\mathrm{C}_{2} \mathrm{H}_{4} \mathrm{OH}\right)$ is insoluble in $\mathrm{CCl}_{4}$, so that a comparison cannot be made. However, similar to HTPA, ${ }^{48}$ the IRRA spectrum of the TDAHA monolayer reveals a single band at $3312 \mathrm{~cm}^{-1}$, indicating that the NH groups of the TDAHA monolayer are also trans oriented and $\mathrm{H}$-bonded. The absence of the free $v(\mathrm{NH})$ peak at $3462 \mathrm{~cm}^{-1}$ proves that all $\mathrm{NH}$ groups in the condensed monolayer phase are involved in H-bonds.

The existence of an extended hydrogen bonding network is supported by the positions of the strong amide I $\left(1640 \mathrm{~cm}^{-1}\right)$ and weak amide II $\left(1552 \mathrm{~cm}^{-1}\right.$ ) bands (Figure 8). Interestingly, the $\mathrm{CH}_{2}$ deformation band appears as a single peak at $1468 \mathrm{~cm}^{-1}$ in the oblique phase below the phase transition, and is split into

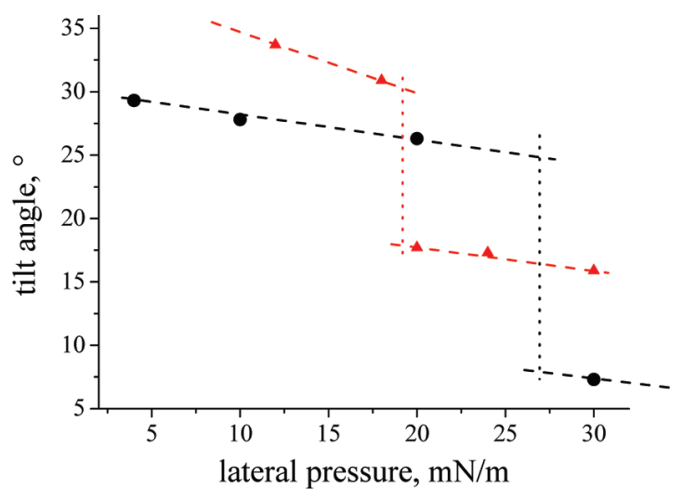

Figure 9. Tilt angle $t$ as a function of the lateral pressure of TDAHA (, at $\left.5^{\circ} \mathrm{C}\right)$ and $\operatorname{HTPA}\left(\boldsymbol{\Delta}\right.$, at $\left.2^{\circ} \mathrm{C}\right)$. The dashed lines are only to guide the eye.

two bands ( 1464 and $1473 \mathrm{~cm}^{-1}$ ) in the $\mathrm{L}^{\prime}$ phase because of the packing in an orthorhombic unit. This is again in perfect accordance with the above-described GIXD data.

The comparison of the two amphiphiles TDAHA and HTPA, ${ }^{48}$ which differ only in the chemical structure by the positions of the two substituents at the acid amide group, reveals clear differences in the main interfacial characteristics. Generally, the oblique phases of TDAHA and HTPA at lower pressures are very similar. This concerns particularly the similar temperature dependence of the tilt angle (Figure 9), but HTPA has a slightly larger crosssectional area and slightly larger tilt angles than TDAHA. However, the tilt angle decrease at the second first-order transition is larger for TDAHA compared to that that of HTPA. The crucial difference exists between these two amphiphiles TDAHA and HTPA in the structure of the second condensed phase observable after the second first-order phase transition at lower temperatures and higher pressures. After the second phase transition, TDAHA changes the structure from oblique to NNN tilted orthorhombic $\left(\mathrm{L}_{2}{ }^{\prime}\right)$ phase, whereas HTPA forms another oblique phase.

\section{CONCLUSION}

Naturally occurring and synthetic amphiphilic $N$-acylethanolamines have attracted particular attention because of a number of interesting biological and medicinal properties. In this respect, the interfacial characteristics of selected compounds can provide useful information.

N-Myristoylethanolamine (tetradecanoic acid-(2-hydroxyethyl)amide, TDAHA) of high purity has been synthesized to obtain information about the main characteristics of its Langmuir monolayers, such as surface pressure-area $(\pi-A)$ isotherms, morphology of the condensed phase domains, lattice structure of the condensed phase, and existence of hydrogen bonds ($\mathrm{NH} \cdot \mathrm{O}=\mathrm{C}-$ ).

The experimental $\pi-A$ isotherms of the TDAHA monolayers spread on pure water and measured in the temperature region between 10 and $36^{\circ} \mathrm{C}$ are well matched to those of monolayers of the usual amphiphiles. The sharp inflection point followed by the plateau region indicates the main first-order phase transition from the fluid phase to the condensed phase. However, at temperatures of $\leq 10^{\circ} \mathrm{C}$, a second inflection point at high surface pressures followed by only a small area change indicates the existence of a second phase transition between two condensed phases. This transition pressure increases strongly with increasing temperature, different from 3-hydroxy- $N$-tridecyl propanoic 
acid amide (HTPA), ${ }^{48}$ which differs only in the chemical structure by exchanging the positions of the two substituents at the acid amide group, where the transition pressure of the second phase transition is nearly independent of temperature. Consequently, the second phase transition of TDAHA monolayers cannot be seen in the $\pi-A$ isotherms above $10{ }^{\circ} \mathrm{C}$, since the monolayer stability does not allow compression above $40 \mathrm{mN} / \mathrm{m}$.

The two-dimensional Clausius-Clapeyron equation has been used to determine the change in enthalpy and entropy for the phase transitions of the monolayer using the slope of the $d \pi_{c} / d T$ curve. The second transition at higher pressures is a very weak firstorder transition with only $10 \%$ of the entropy change observed for the first first-order main phase transitions at the same temperature.

On the mesoscopic scale, the domain shape, characterized by fractal nature with a limited tendency to branching and the absence of an inner texture in the homogeneously reflecting domains, indicates a certain fluidity of the condensed phase.

The characteristic features of the two-dimensional lattice structure were obtained by GIXD studies. Three diffraction peaks observed after the main phase transition indicate an oblique lattice with a cross-sectional area of $19.3 \AA^{2}$. At temperatures of $\leq 10{ }^{\circ} \mathrm{C}$, two diffraction peaks observed after the second phase transition at high pressures reveal a $\mathrm{L}_{2}^{\prime}$ phase characterized by an orthorhombic structure with NNN tilted alkyl chains, distortion in NN direction, and a decrease of the cross-sectional area to $18.8 \AA^{2}$. Whereas in the oblique phase the tilt angle with respect to the surface normal decreases only marginally with increasing pressure, the transition into the orthorhombic $\mathrm{L}_{2}^{\prime}$ phase leads to a drastic decrease of the tilt angle by almost $20^{\circ}$.

The IRRAS experiments confirm the existence of an extended hydrogen bonding network $(-\mathrm{NH} \cdots \mathrm{O}=\mathrm{C}-)$ in the TDAHA monolayers supported by the positions of the strong amide I $\left(1640 \mathrm{~cm}^{-1}\right)$ and weak amide II $\left(1552 \mathrm{~cm}^{-1}\right)$ bands. Furthermore, the $\mathrm{CH}_{2}$ deformation band as a single peak at $1468 \mathrm{~cm}^{-1}$ in the oblique phase below the phase transition and its split into two bands ( 1464 and $1473 \mathrm{~cm}^{-1}$ ) in the L2' phase because of the packing in an orthorhombic unit cell are in perfect accordance with the GIXD results.

The comparison of the two amphiphiles TDAHA and HTPA ${ }^{48}$ reveals crucial differences in the structure of the second condensed phase observable after the second first-order phase transition at lower temperatures and higher pressures. Above this transition, TDAHA changes the monolayer structure from oblique to NNN tilted orthorhombic $\left(\mathrm{L}_{2}{ }^{\prime}\right)$, whereas HTPA forms another oblique phase.

\section{AUTHOR INFORMATION}

\section{Corresponding Author}

*E-mail: dieter.vollhardt@mpikg.mpg.de.

\section{ACKNOWLEDGMENT}

We thank HASYLAB at DESY, Hamburg, Germany, for beamtime and excellent support. This work was supported by the Max Planck Society.

\section{REFERENCES}

(1) Schmid, H. H. O.; Schmid, P. C.; Natarajan, V. Prog. Lipid Res. 1990, 29, 1.
(2) Hansen, H. S.; Moesgaard, B.; Hansen, H. H.; Petersen, G. Chem. Phys. Lipids 2000, 108, 135.

(3) Schmid, H. H. O.; Schmid, P. C.; Natarajan., V. Chem. Phys. Lipids 1996, 80, 133.

(4) Schmid, H. H. O.; Schmid, P. C.; Berdyshev, E. V. Chem. Phys. Lipids 2002, 121, 111.

(5) Devane, W. A.; Hanus, L.; Breuer, A.; Pertwee, R. G.; Stevenson, L. A.; Griön, G.; Gibson, D.; Mandelbaum, A.; Etinger, A; Mechoulam, R. Science 1992, 258, 946.

(6) Schuel, H.; Goldstein, E.; Mechoulam, R.; Zimmerman, A. M.; Zimmerman, S. Proc. Natl. Acad. Sci. U.S.A. 1994, 91, 7678.

(7) Venance, L.; Piomelli, D.; Glowinski, J.; Giaume, C. Nature 1995, 376, 590.

(8) Sugita, M.; Williams, M.; Dulaney, J. T.; Moser, H. W. Biochim. Biophys. Acta 1975, 398, 125.

(9) Chapman, K. D.; Tripathy, S.; Venables, B.; Desouza, A. D. Plant Physiol. 1998, 116, 1163.

(10) Cravatt, B. F.; Lichtman., A. H. Curr. Opin. Chem. Biol. 2003, 7, 469.

(11) Sun, Y.-X.; Tsuboi, K.; Okamoto, Y.; Tonai, T.; Murakami, M.; Kudo, I.; Ueda, N. Biochem. J. 2004, 380, 749.

(12) Domingo, J. C.; Mora, M.; de Madariaga, M. A. Biochim. Biophys. Acta 1993, 1148, 308.

(13) Mercadal, M.; Domingo, J. C.; Bermudez, M.; Mora, M.; de Madariaga, M. A. Biochim. Biophys. Acta 1995, 1235, 281.

(14) Ambrosini, A.; Bertoli, E.; Mariani, P.; Tanfani, F.; Wozniak, M.; Zolese, G. Biochim. Biophys. Acta 1993, 1148, 351.

(15) Rudert, R.; Wu, Y.; Vollhardt, D. Z. Kristallogr. 1996, 211, 114.

(16) Ramakrishnan, M.; Swamy, M. J. Biochim. Biophys. Acta 1999, 1418,261

(17) Kamlekar, R. K.; Swamy, M. J. J. Lipid Res. 2006, 47, 1424.

(18) Berg, J. M.; Tymoczko, J. L.; Stryer, L. Biochemistry, 5th ed.; W. H. Freeman \& Co.: New York, 2003; p 324.

(19) Nelson, D. L.; Cox, M. M. Lehninger, Principles of Biochemistry, 4th ed.; W. H. Freeman \& Co.: New York, 2004; p 353.

(20) Ohanian, J.; Ohanian, V. Cell. Mol. Life Sci. 2001, 58, 2053.

(21) Melzer, V.; Vollhardt, D. Phys. Rev. Lett. 1996, 76, 3770.

(22) Vollhardt, D.; Melzer, V. J. Phys. Chem. B 1997, 101, 3370.

(23) Melzer, V.; Weidemann, G.; Vollhardt, D.; Brezesinski, G.; Wagner, R.; Struth, B.; Möhwald, H. J. Phys. Chem. B 1997, 101, 4752.

(24) Melzer, V.; Weidemann, G.; Vollhardt, D.; Brezesinski, G.; Wagner, R.; Struth, B.; Möhwald, H. Supramol. Sci. 1997, 4, 391.

(25) Melzer, V.; Vollhardt, D. Prog. Colloid Polym. Sci. 1997, $105,1130$.

(26) Melzer, V.; Vollhardt, D.; Brezesinski, G.; Möhwald, H. J. Phys. Chem. B 1998, 102, 591.

(27) Melzer, V.; Vollhardt, D.; Weidemann, G.; Brezesinski, G.; Wagner, R.; Möhwald, H. Phys. Rev. E 1998, 57, 901.

(28) Vollhardt, D.; Melzer, V.; Fainermann, V. B. Thin Solid Films 1998, 327-329, 842 .

(29) Melzer, V.; Vollhardt, D.; Brezesinski, G.; Möhwald, H. Thin Solid Films 1998, 327-329, 857.

(30) Melzer, V.; Weidemann, G.; Wagner, R; Vollhardt, D.; DeWolf, C.; Brezesinski, G.; Möhwald, H. Chem. Eng. Technol. 1998, 21, 44.

(31) Fainerman, V. B.; Vollhardt, D. J. Phys. Chem. B 2003, 107,3098

(32) Vollhardt, D.; Wagner, R. J. Phys. Chem. B 2006, 110, 14881-14889.

(33) Vollhardt, D; Emrich, G. Colloids Surf., A 2000, 161, 173.

(34) Vollhardt, D.; Brezesinski, G.; Siegel, S.; Emrich, G. J. Phys. Chem. B 2001, 105, 12061.

(35) Meunier, J. Colloids Surf., A 2000, 171, 33.

(36) Hönig., D.; Möbius, D. J. Phys. Chem. 1991, 95, 4590.

(37) Vollhardt, D. Adv. Colloid Interface Sci. 1996, 64, 143.

(38) Als-Nielsen, J.; Jaquemain, D; Kjaer, K.; Lahav, M.; Levellier, F.; Leiserowitz, L. Phys. Rep. 1994, 246, 251.

(39) Kjaer, K. Physica B 1994, 198, 100.

(40) Kaganer, V. M.; Möhwald, H.; Dutta, P. Rev. Mod. Phys. 1999, 71,779 . 
(41) Lepere, M.; Chevallard, C.; Brezesinski, G.; Goldmann, M.; Guenoun, P. Angew. Chem., Int. Ed. 2009, 48, 5005-5009.

(42) Mendelsohn, R.; Brauner, J. W.; Gericke, A. Annu. Rev. Phys. Chem. 1995, 46, 305.

(43) Flach, C. R.; Gericke, A.; Mendelsohn, R. J. Phys. Chem. B 1997, 101, 58.

(44) Andreeva, T. D.; Petrov, J. G.; Brezesinski, G.; Möhwald, H. Langmuir 2008, 24, 8001.

(45) Muenter, A. H.; Hentschel, J.; Börner, H. G.; Brezesinski, G. Langmuir 2008, 24, 3306.

(46) Fainerman, V. B.; Vollhardt, D.; Melzer, V. J. Chem. Phys. 1997, 107, 243.

(47) Kitaigorodskii, A. I. Organic Chemical Crystallography; Consultants Bureau: New York, 1961.

(48) Brezesinski, G.; Stefaniu, C.; Nandy, S.; Dutta Banik, D.; Nandi, N.; Vollhardt, D. J. Phys. Chem. C 2010, 114, 15695. 